\title{
70\%-Damped Spectral Acceleration as a Ground Motion Intensity Measure for Predicting Highly Nonlinear Response of Structures
}

\author{
Kenny Buyco, ${ }^{\text {a) }}$ M.EERI, and Thomas H. Heaton, ${ }^{\text {a) }}$ M.EERI
}

\begin{abstract}
We investigate $70 \%$-damped spectral acceleration, $S a^{70 \%}(T)$, as a ground motion intensity measure for predicting maximum interstory drift ratios of $0.03,0.06$, and 0.1 as well as collapse. We perform incremental dynamic analysis with 50 ground motions on 22 steel moment frame building models with heights of 3, 9, and 20 stories. We find that if $T_{1} \leq T \leq 2 T_{1}, S^{70 \%}(T)$ is efficient and usually sufficient for the considered levels of highly nonlinear response. $S a^{70 \%}\left(1.5 T_{1}\right)$ is generally an efficient choice. We find that $S a^{70 \%}(T)$ is similar to average spectral acceleration, $S a_{\text {avg }}$, in many ways, as both intensity measures emphasize a wide range of periods in a ground motion when compared to $S a^{5 \%}\left(T_{1}\right) . S a^{70 \%}(T)$ is equivalent to the peak of a ground motion's low-pass filtered acceleration, and this interpretation may be useful for estimating the potential of a ground motion to elicit a highly nonlinear response. [DOI: 10.1193/ $111417 \mathrm{EQS} 237 \mathrm{M}]$
\end{abstract}

\section{INTRODUCTION}

A ground motion intensity measure (IM) quantifies the intensity of shaking of a ground motion and can have a multitude of applications in earthquake engineering. One application is the performance assessment of buildings to seismic hazards, such as in FEMA P-58 (Federal Emergency Management Agency 2012). The underlying methodology for seismic performance assessment in FEMA P-58 can be expressed, with some simplifications, as the integral in Equation 1 (Moehle and Deierlein 2004, Luco and Cornell 2007):

$$
\lambda[E D P]=\int_{I M} f[E D P \mid I M] \lambda[I M] d[I M]
$$

where engineering demand parameter (EDP; e.g., maximum interstory drift ratio), $f[x \mid y]$ is a probability density function denoting the density of probability that $x$ will be exceeded given $y$, and $\lambda[x]$ is a function representing the mean frequency of exceeding $x$ over some time interval. Thus for a structure at a given site, Equation 1 relates the seismic hazard, defined by some user-selected IM, at that site to the mean frequency of some given EDP being exceeded for the structure. In this formulation, $f[E D P \mid I M]$ is often estimated by performing time-history analysis with a mathematical model of the specific structure when subjected to a

\footnotetext{
a) California Institute of Technology, 1200 E. California Boulevard, Pasadena, CA 91125; Email: kenny.buyco@arup.com (K. B.)
} 
suite of ground motions. In many applications, the distribution corresponding to $f[E D P \mid I M]$ is assumed to be lognormal, in which case, only a median (or, equivalently for a lognormal distribution, geometric mean) and lognormal standard deviation, $\sigma_{\ln }$, are needed to develop $f[E D P \mid I M]$.

In selecting an appropriate IM, it is important to evaluate its efficiency and sufficiency (Luco and Cornell 2007) regarding $f[E D P \mid I M]$. An IM is efficient if $\sigma_{\ln }$ associated with $f[E D P \mid I M]$ is low. A more efficient IM reduces the number of ground-motion time-history analyses necessary to estimate $f[E D P \mid I M]$ to a given level of confidence, reducing the computation time necessary for performance evaluation. An IM is sufficient if $f[E D P \mid I M]$ is not dependent on ground motion characteristics other than the IM, such as earthquake magnitude. A more sufficient IM broadens the set of ground motions that can be used in analyses, as other factors do not need to be considered when estimating $f[E D P \mid I M]$. Note that the evaluation of efficiency and sufficiency for a given IM will depend on the EDP or EDPs of interest.

In Equation 1, calculation of $\lambda[I M]$ requires seismic hazard analysis at the given site. This is commonly done using probabilistic seismic hazard analysis, which requires the development of ground motion prediction equations (GMPEs; e.g., Boore and Atkinson 2008), for the chosen IM. For practicality, some researchers restrict choices of IMs to those for which $\lambda[I M]$ can be readily calculated using existing GMPEs, but we do not consider that restriction in this study.

In practice, the most common IM used in design and performance assessment is $5 \%$-damped (pseudo) spectral acceleration, calculated at the fundamental period $\left(T_{1}\right)$ of the building of interest. Spectral acceleration with damping ratio, $\zeta$, and period, $T$, will be denoted in this paper as $S a^{\zeta}(T)$. Note that in most applications, the damping ratio, $\zeta$, is dropped from the notation of spectral acceleration because the value of 5\% is so prevalent. As such, 5\%-damped spectral acceleration is often denoted $S a(T)$, with $\zeta=5 \%$ implicitly assumed. In general, $S a^{\zeta}(T)$ is calculated for a ground acceleration, $\ddot{u}_{g}(t)$, according to Equation 2:

$$
S a^{\zeta}(T)=\omega^{2} \max _{t}|u(t)|
$$

where $u(t)$ is the solution to Equation 3:

$$
\ddot{u}(t)+2 \zeta \omega \dot{u}(t)+\omega^{2} u(t)=-\ddot{u}_{g}(t),
$$

$\omega=2 \pi / T$, and $t$ is time. Often, the response spectrum of a ground motion will be generated, which is simply a plot of $S a^{\zeta}(T)$ versus $T$. Physically, $S a^{\zeta}(T)$ approximately represents the maximum acceleration experienced by a $\zeta$-damped single-degree-of-freedom (SDOF) system with period, $T$, when it is subjected to the ground acceleration, $\ddot{u}_{g}(t)$.

Within the modern framework for developing and evaluating IMs, $S a^{5 \%}\left(T_{1}\right)$ has been shown to generally be an efficient IM (Shome et al. 1998), but there is still significant room for improvement, particularly regarding its efficiency and sufficiency when the EDP represents a highly nonlinear response. Modern improvements to $S a^{5 \%}\left(T_{1}\right)$ account for the "spectral shape" of a ground motion's 5\%-damped response spectrum in some manner. Spectral shape describes $S a^{5 \%}(T)$ of a ground motion over some range of $T$, often at periods longer than $T_{1}$ and sometimes at the natural periods of vibration of higher modes (i.e., $T_{2}, T_{3}$, etc.). 
Periods longer than $T_{1}$ are especially important when considering nonlinear structural response because a building will experience "period-lengthening" as it accumulates damage. Haselton and Baker (2006) showed that simply using $S a^{5 \%}\left(2 T_{1}\right)$ instead of $S a^{5 \%}\left(T_{1}\right)$ is a more efficient IM for collapse predictions. Several researchers (e.g., Cordova et al. 2000, Vamvatsikos and Cornell 2005, Bianchini et al. 2009, Eads et al. 2015) have found that taking the average of $S a^{5 \%}(T)$ calculated at multiple periods can be an effective IM. This IM is often referred to as "average spectral acceleration" and is denoted $S a_{\text {avg }}$ in this paper.

Baker and Cornell (2005) demonstrated that $\varepsilon\left(T_{1}\right)$ can be used to indirectly measure spectral shape, and its inclusion in GMPEs makes it convenient to include in seismic risk analysis. $\varepsilon\left(T_{1}\right)$ of a ground motion record is the number of standard deviations that the recorded $S a^{5 \%}\left(T_{1}\right)$ is greater than what a user-selected GMPE would predict as the median expected $S a^{5 \%}\left(T_{1}\right)$ given the source and site characteristics for the record. To date, $\varepsilon\left(T_{1}\right)$, when used in conjunction with $S a^{5 \%}\left(T_{1}\right)$, is the most common measure of spectral shape in practice.

In this study, we evaluate a new IM, $S a^{70 \%}(T)$, and evaluate its efficiency and sufficiency for predicting a highly nonlinear response. We define highly nonlinear response as maximum interstory drift ratios (MIDRs) in excess of 0.03 , with special attention paid to the following EDPs: MIDR $=0.03$, MIDR $=0.06$, MIDR $=0.1$, and collapse. Song and Heaton (2012) first introduced peak filtered acceleration, defined to be the peak value of the acceleration ground motion after it is low-pass filtered, as an IM for predicting collapse. Coincidentally, if the lowpass filter is a second-order Butterworth filer and the corner period is $T$, then the peak filtered acceleration of a ground motion is exactly equivalent to $S a^{1 / \sqrt{2}}(T)$ (Song 2013). In this study, we choose $\mathrm{Sa}^{70 \%}(T)$ as the IM instead of peak filtered acceleration because $1 / \sqrt{2} \approx 70 \%$ and the concept of spectral acceleration has a long history in earthquake engineering.

It may be surprising that spectral acceleration calculated with $\zeta$ as high as $70 \%$ could be an effective IM considering researchers have found the effective damping of actual structures in earthquakes to be around 5\% (Haviland 1976), with some finding the effective damping of severely damaged structures to be in the range of 10\%-20\% (e.g., Iwan and Gates 1979, Guyader 2004). To briefly justify the use of high damping, consider Equation 3. If $\zeta \rightarrow \infty$, then Equation 3 simplifies to:

$$
u(t) \approx-(1 / 2 \zeta \omega) \dot{u}_{g}(t)
$$

Inserting into Equation 2 yields:

$$
\lim _{\zeta \rightarrow \infty} S a^{\zeta}(T)=\omega /(2 \zeta) \max _{t}\left|\dot{u}_{g}(t)\right| \propto P G V,
$$

where PGV is the ground motion's peak ground velocity. Constant scaling does not change the effectiveness of an IM, so $\lim _{\zeta \rightarrow \infty} S a^{\zeta}(T)$ is equivalent to PGV as an IM, regardless of $T$. PGV has been shown to be an effective IM for predicting nonlinear response (Akkar and Ozen 2005), and, in fact, it is used in Japan as the IM when scaling ground motions to a specified hazard in performance-based seismic design of high-rise buildings (Masayoshi et al. 2012). This leads to the somewhat surprising conclusion that $\lim _{\zeta \rightarrow \infty} S a^{\zeta}(T)$ must also 
be an effective IM for predicting nonlinear response despite the fact that $\zeta \rightarrow \infty$ is clearly not a physically realistic measure of the effective damping of any structure. As such, that previous research has indicated that $\zeta=70 \%$ is not physically representative of the effective damping of structures does not preclude the possibility that $\operatorname{Sa}^{70 \%}(T)$ is an effective IM.

In this study, we consider 22 steel moment-resisting frame building models and perform incremental dynamic analysis (IDA) on each building model with a set of 50 recorded ground motions. We evaluate the efficiency and sufficiency of $\mathrm{Sa}^{70 \%}(T)$ at predicting $\mathrm{MIDR}=0.03$, $\mathrm{MIDR}=0.06, \mathrm{MIDR}=0.1$, and collapse and compare it to other IMs, with an emphasis on $S a^{5 \%}\left(T_{1}\right)$ and $S a_{\text {avg }}$.

\section{MODELING CONSIDERATIONS}

\section{BUILDING MODELS}

The building designs we consider in this study were developed for the SAC Joint Venture by Gupta and Krawinkler (1999) and Lee and Foutch (2000), who designed 3-, 9-, and 20-story steel moment frame buildings based on the 1973, 1985, and 1994 Uniform Building Code (UBC). Section and material properties are available in the aforementioned citations. The 1973 UBC does not include seismic drift provisions, but in practice, wind drift limits were sometimes applied in seismic design. Thus we consider two designs with and without drift limits according to the 1973 UBC for the 3- and 9-story configurations. There is only one 1973 UBC 20-story design because the wind drift limit controlled its member properties. In total, we consider four 3-story, four 9-story, and three 20-story building designs.

We create two-dimensional finite element models of each building using Frame-2d, a computer program that is specifically designed to calculate the seismic response of steel moment frame and braced frame buildings by using fiber elements to model the behavior of beams and columns. The cross section of each element is divided into fibers that have hysteretic axial stress-strain relationships, equipped with a yield plateau and strain-hardening or softening region. Geometric nonlinearities (e.g., P- $\Delta$ ) are accounted for by updating the nodal positions at each time step. Challa (1992), Challa and Hall (1994), Hall and Challa (1995), and Hall (1998) validated the special features of Frame-2d, such as joint modeling, nodal updating, and weld fracture, by extensive numerical testing and comparison with experimental data. Boundary conditions, gravity loads, and seismic masses are applied in accordance with the modeling conducted by Gupta and Krawinkler (1999).

For each of the 11 building designs, we develop two building models: one with "perfect" moment connections ("P model") and one with pre-Northridge "brittle" moment connections ("B model"). The brittle connections model the failures of welded moment connections observed after the 1994 Northridge earthquake. In B models, the short fibers at the end of beam elements that are connected to columns via welded moment connections represent weld fibers and are assigned a random fracture strain according to a user-defined probability distribution. The fracture distributions used in this study are the same as those used by Krishnan and Muto (2012) and similar to those used by Hall (1998), which were calibrated to weld fracture observations in the Northridge earthquake.

We perform pushover analysis for all 22 building models considered in this study, calculated according to the procedure described by Hall (1997). The vertical distribution 
Table 1. Building model characteristics

\begin{tabular}{lccc}
\hline \hline & & \multicolumn{2}{c}{$V_{\max } / W$} \\
\cline { 3 - 4 } Design & $T_{1}$ (sec.) & P model & B model \\
\hline 3-94 & 0.78 & 0.433 & 0.340 \\
3-85 & 0.94 & 0.282 & 0.242 \\
3-73wD & 0.84 & 0.360 & 0.298 \\
3-73noD & 1.01 & 0.226 & 0.211 \\
9-94 & 1.88 & 0.180 & 0.115 \\
9-85 & 2.16 & 0.126 & 0.089 \\
9-73wD & 1.83 & 0.179 & 0.117 \\
9-73noD & 2.72 & 0.078 & 0.065 \\
20-94 & 3.50 & 0.085 & 0.062 \\
20-85 & 3.21 & 0.096 & 0.064 \\
20-73 & 3.10 & 0.101 & 0.066 \\
\hline \hline
\end{tabular}

of loads are proportioned in accordance with equivalent first-mode seismic design loads (American Society of Civil Engineers 2010). Buyco (2018) provides a more detailed description of the methods and results of pushover analysis for these models. To summarize, Table 1 shows the maximum base shear $\left(V_{\max }\right)$, defined as the maximum of the pushover curve, normalized by the seismic weight $(W)$ for each building model along with $T_{1}$. The B models are nondeterministic because the fracture strains are randomly assigned according to a probability distribution, so the reported $V_{\max } / W$ values are the mean of three pushover analyses with different fracture strain assignments that were generated independently. As expected, every B model has a lower $V_{\max } / W$ than its corresponding $\mathrm{P}$ model. Each design is denoted first by the number of stories (3, 9, or 20) and then by the design year $(94,85$, or 73$)$. The 1973 UBC designs that did and did not incorporate seismic drift limits are denoted "wD" and "noD," respectively.

\section{IDA}

The set of 50 ground motions we use in this study are from the ground motion record sets generated in ATC-63 for collapse assessment of building structures (Federal Emergency Management Agency 2009). These processed recorded ground motion records are available in the PEER NGA-West2 Database (Pacific Earthquake Engineering Research Center 2013). The records span a magnitude range of $6.50 \leq M \leq 7.90$ and a source-to-site Joyner-Boore distance (kilometers) range of $0.0 \leq R \leq 26.0$. Out of the 50 ground motions, 28 are nearsource $(R<10 \mathrm{~km})$. ATC-63 classifies half of these near-source records as containing pulses.

We conducted IDA (Vamvatsikos and Cornell 2002) on all 22 building models with all 50 biaxial ground motions. We do not consider vertical ground motion. We perform IDA for each building model and ground motion for both horizontal components by multiplying the ground motion by a scale factor and performing the dynamic analysis. The scale factor on the input ground motion starts at 0.05 and increments by 0.05 for each successive analysis. We recorded the MIDR for each dynamic analysis. For a given biaxial ground motion, scale 
factor, and building model, we take the corresponding MIDR to be the maximum of the two MIDRs computed for the two horizontal components. When calculating an IM for a ground motion, we use the horizontal component for which the MIDR of interest is first achieved.

We investigate three main MIDR values as EDPs in this study to represent highly nonlinear response: $0.03,0.06$, and 0.1 . MIDR $=0.03$ is chosen because it is the collapseprevention limit for many performance-based applications (e.g., Tall Buildings Initative Guidelines Working Group 2010, Los Angeles Tall Buildings Structural Design Council 2015). MIDR $=0.06$ is approximately the ultimate limit of modern ductile moment connections (American Institute of Steel Construction 2010), at which point, failure because of local flange buckling may occur. This is an effect that cannot be captured by Frame-2d. $\mathrm{MIDR}=0.1$ corresponds to a severely damaged building and is considered by some to be the default global collapse limit (Lee and Foutch 2000). We also consider "collapse" as an EDP, defined in this study as the point at which the building model succumbs to P- $\Delta$ collapse in simulation, indicated by numerical instability. It should be noted that other EDPs (e.g., peak floor acceleration and local interstory drift ratio) are important for evaluating structural response but are not considered here.

Recall that for each building height (3-, 9-, or 20-story), there are three or four different designs. Additionally, each building design has a $\mathrm{P}$ model and a $\mathrm{B}$ model. In this paper, we specify an individual building model by its height, weld model type, and design year in that order. For example, the 3-story P model designed according to $1985 \mathrm{UBC}$ is abbreviated as the "3P-85" model. Furthermore, we often combine statistics for all models with the same height and weld model type together and abbreviate, for example, the four 3-story P models as "3P" models.

\section{EFFICIENCY OF $\mathrm{Sa}^{70 \%}(\mathrm{~T})$}

In this section, we evaluate the efficiency of $\mathrm{Sa}^{70 \%}(\mathrm{~T})$ and compare it with other IMs. To calculate the efficiency for a given EDP, IM, and building model, we compute the lognormal standard deviation $\left(\sigma_{\ln }\right)$ of the IM values of the 50 scaled ground motions that elicit the EDP in the building model. $\sigma_{\ln }$ measures the dispersion of the IM values that produce the same response, so a more efficient IM will have a low $\sigma_{\mathrm{ln}}$. For a given EDP, IM, and set of building models (e.g., the four 3P models), $\sigma_{\ln }$ is evaluated on the residual of individual IM values after normalizing by the geometric mean of the IM values for the particular building model. This normalization is done so that IM values from analyses of different building models can be combined for the calculation of $\sigma_{\ln }$.

\section{EVALUATING EFFICIENCY OF $\operatorname{Sa}^{\zeta}(T)$ FOR ALL $T$ AND $\zeta$}

To demonstrate how the most efficient choices of $T$ and $\zeta$ for $S a^{\zeta}(T)$ change as MIDR increases, we first consider the 3P models, whose responses are first-mode dominated. For MIDRs from 0.005 to 0.1 , we calculate $\sigma_{\ln }=\sigma_{\ln }(T, \zeta)$ of $S a^{\zeta}(T)$, with $\zeta$ ranging from $1 \%$ to $200 \%$ and $T$ ranging from $0.5 T_{1}$ to $2.5 T_{1}$. Then for each MIDR we calculate the following:

$$
\sigma_{\mathrm{ln}, \min }=\min _{T, \zeta} \sigma_{\ln }(T, \zeta)
$$




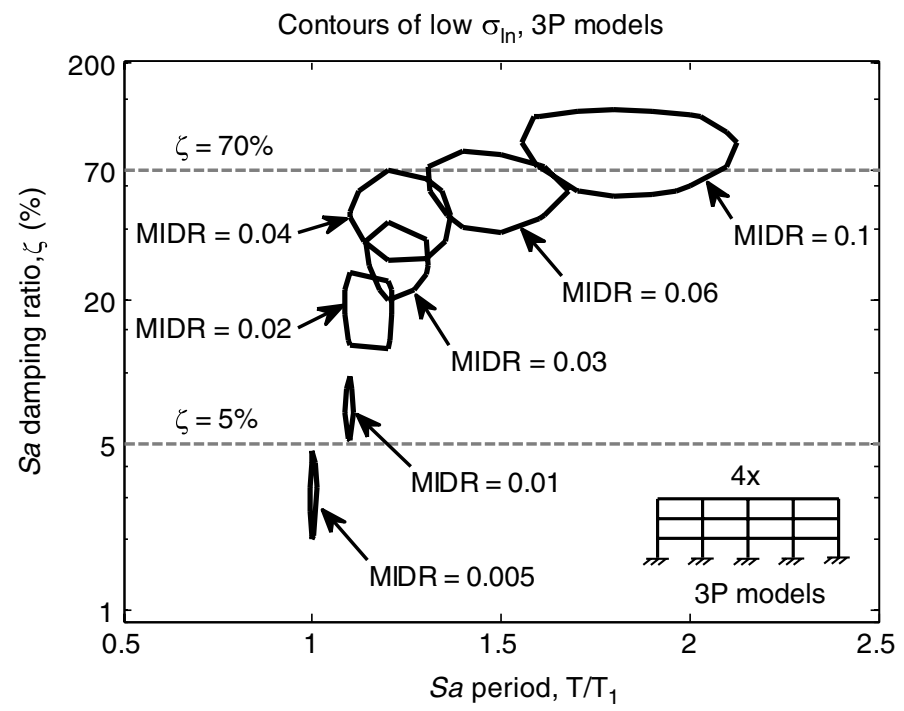

Figure 1. Contours representing $\sigma_{\ln } \leq 1.05 \sigma_{\ln , \min }$ of $S a^{\zeta}(T)$ for each considered MIDR, ranging from 0.005 to 0.1 . Results from the $3 \mathrm{P}$ models are shown here.

To visualize the $(T, \zeta)$ pairs that produce the most efficient $S a^{\zeta}(T)$, we plot in Figure 1 a contour for each MIDR such that each contour encloses the set of $(T, \zeta)$ pairs for which $\sigma_{\mathrm{ln}} \leq 1.05 \sigma_{\mathrm{ln}, \min }$. Each contour area thus represents a region of low $\sigma_{\mathrm{ln}}$, for which choosing any $(T, \zeta)$ pair in that region will achieve nearly the minimum possible $\sigma_{\ln }$ for the associated MIDR. For the 3P models, the regions of low $\sigma_{\mathrm{ln}}$ exhibit a clear trend as MIDR increases. For moderate drifts $(\mathrm{MIDR}=0.005$ and 0.01 ), the regions are very small and have $\zeta$ less than $10 \%$ with $T \approx T_{1}$. For large drifts (MIDR $=0.02,0.03$, and 0.04 ), the regions move upward to $\zeta$ from $20 \%$ to $70 \%$ and become larger, indicating that many $(T, \zeta)$ pairs can yield low $\sigma_{\ln }$. For extremely large drifts, nearing global collapse (MIDR $\geq 0.06$ ), the regions become even larger and expand to the right, indicating that a large range of $\zeta$ around $70 \%$ and periods around $1.5 T_{1}$ will achieve low $\sigma_{\ln }$ at these drift levels.

We combine statistics from all building models to develop the contours of low $\sigma_{\ln }$ in Figure 2. The two plots in Figure 2 show the regions of low $\sigma_{\ln }$ for MIDR $=0.03$, MIDR $=0.06, \mathrm{MIDR}=0.1$, and collapse for all $11 \mathrm{P}$ models and all $11 \mathrm{~B}$ models. MIDRs lower than 0.03 are not included because higher-mode effects cause distortions in these contours when statistics from the 9- and 20-story models are included. The main observation from these results is that when all $\mathrm{P}$ models and $\mathrm{B}$ models are considered, for nearly all highly nonlinear EDPs (the exception being MIDR $=0.03$ for B models), there exists a range of $T$ between $T_{1}$ and $2 T_{1}$ for which $\mathrm{Sa}^{70 \%}(T)$ has $\sigma_{\ln }$ within $5 \%$ of the $\sigma_{\ln , \min }$. This implies that when $T$ is chosen appropriately, $S a^{70 \%}(T)$ is generally an efficient IM for predicting highly nonlinear MIDR response.

Even though we are specifically investigating $S a^{70 \%}(T)$, it should be noted that $S a^{\zeta}(T)$ calculated with $\zeta$ slightly greater than and less than $70 \%$ also appear to have low $\sigma_{\ln }$, 


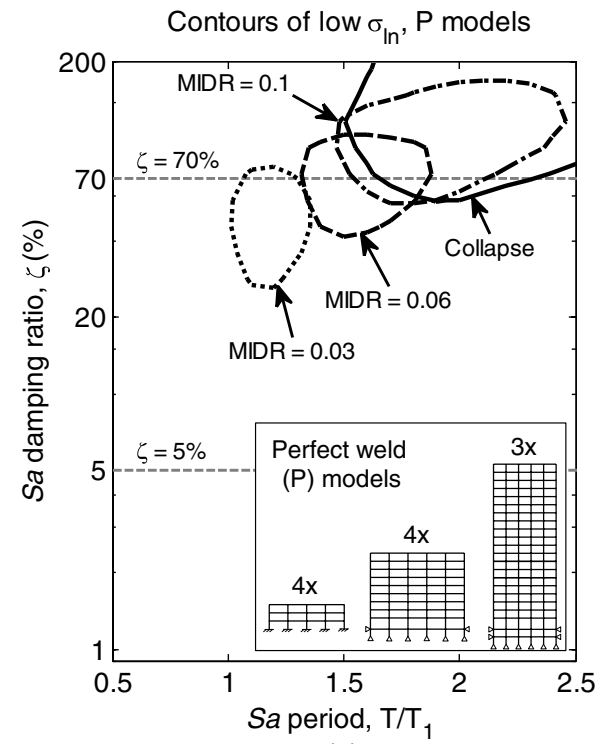

(a)

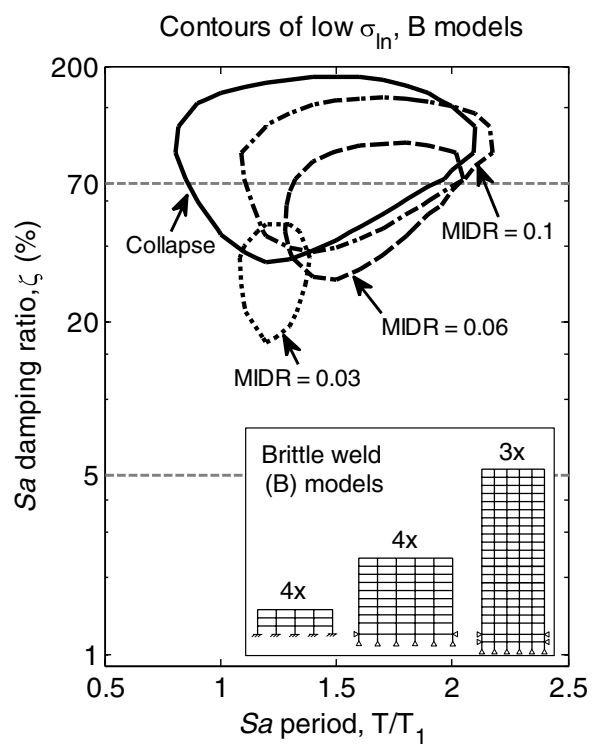

(b)

Figure 2. Contours representing $\sigma_{\ln } \leq 1.05 \sigma_{\ln , \min }$ of $S a^{\zeta}(T)$ for four EDPs: MIDR $=0.03$, MIDR $=0.06, \mathrm{MIDR}=0.1$, and collapse. Results from all of the (a) P and (b) B models are shown.

sometimes lower than $\sigma_{\ln }$ for $\zeta=70 \%$. Despite this, we choose a $\zeta=70 \%$ as the focus of this study for three reasons. First, the difference in $\sigma_{\ln }$ at large drifts between $\operatorname{Sa}^{70 \%}(T)$ and, for example, $\mathrm{Sa}^{50 \%}(T)$ or $S a^{100 \%}(T)$ is generally small when compared with $\sigma_{\ln }$ for $S a^{5 \%}(T)$. This means that, in general, as long as $\zeta$ is high enough, the particular value does not have a large effect on $\sigma_{\mathrm{ln}}$. Second, as previously observed, for nearly all considered highly nonlinear EDPs and building models, $S a^{70 \%}(T)$ has a low $\sigma_{\ln }$ for a range of $T$ between $T_{1}$ and $2 T_{1}$. Even though for a particular building model and EDP there may be a better choice for $\zeta$ that produces a lower $\sigma_{\ln }$, calculating $S a^{\zeta}(T)$ with $\zeta=70 \%$ will give a result that is close to the best for a wide range of building models and EDPs, indicating robustness. Lastly, as described in the introduction, $S a^{70 \%}(T)$ is effectively equivalent to the peak acceleration of a record after it is filtered by a low-pass second-order Butterworth filter, making it a special damping ratio independent of its application to building response. This means the value of $\mathrm{Sa}^{70 \%}(T)$ for a ground motion is physically meaningful, which will be discussed later in this paper.

\section{EFFICIENCY OF $S a^{\zeta}(T)$ COMPARED WITH OTHER IMS}

We compare $\sigma_{\ln }$ of $S a^{70 \%}\left(T_{1}\right), S a^{70 \%}\left(1.5 T_{1}\right)$, and $S a^{70 \%}\left(2 T_{1}\right)$ to that of other ground motion IMs for each set of P models. The IMs compared with $S a^{70 \%}(T)$ are $S a^{5 \%}\left(T_{1}\right)$, $S a^{5 \%}\left(1.5 T_{1}\right), S a^{5 \%}\left(2 T_{1}\right), S a^{20 \%}\left(T_{1}\right), S d_{i}, \mathrm{PGV}$, and three different versions of $S a_{\mathrm{avg}}$. While PGV is a relatively simple IM, $S d_{i}$ and $S a_{\text {avg }}$ are state-of-the-art in terms of predicting a highly nonlinear response. 
We calculate $S d_{i}$ in the same way as $S d^{5 \%}\left(T_{1}\right)$, where $S d^{5 \%}(T)=\left(1 / \omega^{2}\right) S a^{5 \%}(T)$; however, when the displacement exceeds a specified yield point, the simulated SDOF undergoes perfectly plastic inelastic deformation. We calculate the specified yield point based on each building's pushover curve according to Aslani and Miranda (2005) and Applied Technology Council (1996). Luco and Cornell (2007) found $S d_{i}$ to be a more efficient IM than $S a^{5 \%}\left(T_{1}\right)$ for predicting nonlinear response, particularly for low-rise buildings. For a fair comparison, we only consider the P models, as $S d_{i}$ implicitly assumes that the building model is ductile and thus is not well-equipped to predict the response of a building model with brittle welds.

In this paper, we define $S a_{\text {avg }}^{c T_{1}}$ to be the geometric mean $S a^{5 \%}(T)$ of a ground motion with $T$ ranging from $0.2 T_{1}$ to $c T_{1}$ calculated with a period increment of $0.01 \mathrm{sec}$. The optimal upper bound period $c T_{1}$ depends on the application, with larger upper bound periods usually used for more severe EDPs. In this paper, we investigate an upper bound period of $1.5 T_{1}$ because of its use by Kazanti and Vamvatsikos (2015) and Kohrangi et al. (2016) for a wide range of EDPs and an upper bound of $3 T_{1}$ because of its implementation by Eads et al. (2015) for collapse risk assessment. We also consider an upper bound of $2 T_{1}$ because in Chapter 16 ASCE 7-16 (American Society of Civil Engineers 2016), the upper bound of the period range for scaling or matching input ground motions to the $\mathrm{MCE}_{\mathrm{R}}$ response spectrum is required to be at least $2 T_{1}$. A lower bound of $0.2 T_{1}$ is specified in order to incorporate periods as short as $T_{3}$. This lower bound is used in most of the aforementiond citations.

Tables 2-4 show $\sigma_{\ln }$ for all the considered IMs for each set of $\mathrm{P}$ models for MIDR $=0.03$, $\operatorname{MIDR}=0.06, \operatorname{MIDR}=0.1$, and collapse, denoted $\sigma_{\ln , 0.03}, \sigma_{\ln , 0.06}, \sigma_{\ln , 0.1}$, and $\sigma_{\ln , \text { collapse }}$, respectively. For brevity, results for the $\mathrm{B}$ models are not shown, but the observations are generally similar to those made for the $\mathrm{P}$ models. Compared with the other IMs, $\mathrm{Sa}^{70 \%}\left(1.5 T_{1}\right)$ is either the most or nearly the most efficient IM for all $\mathrm{P}$ models and considered EDPs. $\mathrm{Sa}^{70 \%}\left(T_{1}\right)$ is generally efficient for MIDR $=0.03$, even when compared with $S a^{70 \%}\left(1.5 T_{1}\right)$, but does not perform as well for more severe EDPs. The efficiency of

Table 2. $\sigma_{\ln }$ for $3 \mathrm{P}$ models

\begin{tabular}{|c|c|c|c|c|}
\hline IM & $\sigma_{\ln , 0.03}$ & $\sigma_{\ln , 0.06}$ & $\sigma_{\ln , 0.1}$ & $\sigma_{\text {In,collapse }}$ \\
\hline$S a^{5 \%}\left(T_{1}\right)$ & 0.25 & 0.30 & 0.35 & 0.42 \\
\hline$S a^{5 \%}\left(1.5 T_{1}\right)$ & 0.27 & 0.28 & 0.31 & 0.38 \\
\hline$S a^{5 \%}\left(2 T_{1}\right)$ & 0.40 & 0.34 & 0.33 & 0.37 \\
\hline $\mathrm{Sa}^{20 \%}\left(T_{1}\right)$ & 0.17 & 0.24 & 0.31 & 0.37 \\
\hline $\mathrm{Sa}^{70 \%}\left(T_{1}\right)$ & 0.16 & 0.20 & 0.27 & 0.35 \\
\hline$S a^{70 \%}\left(1.5 T_{1}\right)$ & 0.18 & 0.15 & 0.18 & 0.28 \\
\hline$S a^{70 \%}\left(2 T_{1}\right)$ & 0.26 & 0.19 & 0.17 & 0.24 \\
\hline PGV & 0.31 & 0.27 & 0.24 & 0.26 \\
\hline$S d_{i}$ & 0.22 & 0.28 & 0.27 & 0.32 \\
\hline$S a_{\text {avg }}^{1.5 T_{1}}$ & 0.22 & 0.30 & 0.34 & 0.41 \\
\hline$S a_{\mathrm{avg}}^{2 T_{1}}$ & 0.17 & 0.23 & 0.27 & 0.34 \\
\hline$S a_{\text {avg }}^{3 T_{1}}$ & 0.20 & 0.19 & 0.21 & 0.26 \\
\hline
\end{tabular}

Note: The minimum for each EDP is bolded. 
Table 3. $\sigma_{\ln }$ for $9 \mathrm{P}$ models

\begin{tabular}{|c|c|c|c|c|}
\hline IM & $\sigma_{\ln , 0.03}$ & $\sigma_{\ln , 0.06}$ & $\sigma_{\ln , 0.1}$ & $\sigma_{\ln , \text { collapse }}$ \\
\hline$S a^{5 \%}\left(T_{1}\right)$ & 0.35 & 0.38 & 0.40 & 0.41 \\
\hline$S a^{5 \%}\left(1.5 T_{1}\right)$ & 0.41 & 0.34 & 0.37 & 0.39 \\
\hline$S a^{5 \%}\left(2 T_{1}\right)$ & 0.52 & 0.44 & 0.41 & 0.41 \\
\hline$S a^{20 \%}\left(T_{1}\right)$ & 0.27 & 0.30 & 0.34 & 0.36 \\
\hline$S a^{70 \%}\left(T_{1}\right)$ & 0.22 & 0.24 & 0.29 & 0.30 \\
\hline$S a^{70 \%}\left(1.5 T_{1}\right)$ & 0.24 & 0.19 & 0.23 & 0.25 \\
\hline$S a^{70 \%}\left(2 T_{1}\right)$ & 0.29 & 0.21 & 0.22 & 0.25 \\
\hline PGV & 0.27 & 0.27 & 0.26 & 0.28 \\
\hline$S d_{i}$ & 0.27 & 0.27 & 0.28 & 0.31 \\
\hline$S a_{\text {avg }}^{1.5 T_{1}}$ & 0.23 & 0.29 & 0.33 & 0.33 \\
\hline$S a_{\mathrm{avg}}^{2 T_{1}}$ & 0.21 & 0.22 & 0.25 & 0.27 \\
\hline$S a_{\text {avg }}^{3 S_{1}}$ & 0.29 & 0.23 & 0.22 & 0.23 \\
\hline
\end{tabular}

Note: The minimum for each EDP is bolded.

Table 4. $\sigma_{\ln }$ for 20P models

\begin{tabular}{lcccc}
\hline \hline $\mathrm{IM}$ & $\sigma_{\ln , 0.03}$ & $\sigma_{\ln , 0.06}$ & $\sigma_{\ln , 0.1}$ & $\sigma_{\text {ln, collapse }}$ \\
\hline$S a^{5 \%}\left(T_{1}\right)$ & 0.32 & 0.36 & 0.39 & 0.40 \\
$\mathrm{Sa}^{5 \%}\left(1.5 T_{1}\right)$ & 0.43 & 0.37 & 0.40 & 0.41 \\
$\mathrm{Sa}^{5 \%}\left(2 T_{1}\right)$ & 0.56 & 0.50 & 0.52 & 0.52 \\
$\mathrm{Sa}^{20 \%}\left(T_{1}\right)$ & 0.26 & 0.29 & 0.32 & 0.33 \\
$\mathrm{Sa}^{70 \%}\left(T_{1}\right)$ & 0.27 & 0.26 & 0.27 & 0.28 \\
$\mathrm{Sa}^{70 \%}\left(1.5 T_{1}\right)$ & 0.26 & 0.22 & 0.25 & 0.26 \\
$S a^{70 \%}\left(2 T_{1}\right)$ & 0.29 & 0.23 & 0.26 & 0.27 \\
$\mathrm{PGV}$ & 0.38 & 0.30 & 0.30 & 0.30 \\
$S d_{i}$ & $\mathbf{0 . 2 5}$ & 0.26 & 0.29 & 0.30 \\
$S a_{\text {avg }}^{1.5 T_{1}}$ & 0.27 & 0.27 & 0.27 & 0.27 \\
$S a_{\text {avg }}^{2 T_{1}}$ & 0.26 & $\mathbf{0 . 2 1}$ & $\mathbf{0 . 2 3}$ & $\mathbf{0 . 2 4}$ \\
$\mathrm{Sa}_{\text {avg }}^{3 T_{1}}$ & 0.34 & 0.27 & 0.29 & 0.29 \\
\hline \hline
\end{tabular}

Note: The minimum for each EDP is bolded.

$\mathrm{Sa}^{70 \%}\left(2 T_{1}\right)$ is usually very similar to that of $\mathrm{Sa}^{70 \%}\left(1.5 T_{1}\right)$, though it is slightly worse for MIDR $=0.03$. It should be noted that the values of $\sigma_{\ln }$ are relatively similar for $\operatorname{Sa}^{70 \%}\left(T_{1}\right)$, $S a^{70 \%}\left(1.5 T_{1}\right)$, and $S a^{70 \%}\left(2 T_{1}\right)$ for most sets of building models and EDPs, indicating that small inaccuracies in the estimation of $T_{1}$ for a real building will not have a dramatic impact on the efficiency of $\mathrm{Sa}^{70 \%}(\mathrm{~T})$ for highly nonlinear response.

$S a^{5 \%}\left(T_{1}\right), S a^{5 \%}\left(1.5 T_{1}\right)$, and $S a^{5 \%}\left(2 T_{1}\right)$ are the least efficient at almost all EDPs and for all sets of models, showing that they are consistently the worst of the considered IMs for predicting highly nonlinear response. $S d_{i}$ and PGV are more efficient than the standard $S a^{5 \%}\left(T_{1}\right)$ but do not consistently perform better than $S a^{70 \%}\left(1.5 T_{1}\right)$. 
For the different considered versions of $S a_{\mathrm{avg}}, S a_{\mathrm{avg}}^{2 T_{1}}$ is among the most efficient IMs for MIDR $=0.03$ for all P models and for all EDPs for the 20P models. $S a_{\text {avg }}^{3 T_{1}}$ tends to be relatively more efficient for more severe EDPs but does not perform as well for MIDR $=0.03$ and MIDR $=0.06$ compared with other IMs. $S a_{\text {avg }}^{1.5 T_{1}}$ is relatively efficient for MIDR $=0.03$, but never performs better than $S a_{\text {avg }}^{2 T_{1}}$. Of all the considered IMs, regarding efficiency, $S a^{70 \%}\left(1.5 T_{1}\right)$ and $S a_{\text {avg }}^{2 T_{1}}$ seem to be generally the most efficient for a wide range of EDPs representing highly nonlinear response for most of the considered building models. The differences between the two are almost negligible in terms of efficiency, though $\mathrm{Sa}^{70 \%}\left(1.5 T_{1}\right)$ tends to be slightly more efficient for the more severe EDPs (MIDR $\geq 0.06$ ) and for the 3P and 9P models, while $S a_{\text {avg }}^{2 T_{1}}$ tends to be slightly more efficient for MIDR $=0.03$ and for the $20 \mathrm{P}$ models. It should be noted that neither $\mathrm{Sa}^{70 \%}\left(1.5 T_{1}\right)$ nor $S a_{\mathrm{avg}}^{2 T_{1}}$ is the most efficient at all response levels. For a given EDP or building model, $S a^{70 \%}(T)$ and $S a_{\text {avg }}$ can always be finely tuned to find a specific $T$ or period range that gives the best results. Furthermore, $\sigma_{\ln }$ never reaches zero in any case in Tables $2-4$, indicating that there is always some record-to-record variability, even for the most efficient IMs.

\section{SUFFICIENCY OF $\mathrm{Sa}^{70 \%}(\mathrm{~T})$}

In this section, we measure the sufficiency of $S a^{70 \%}\left(1.5 T_{1}\right)$ with respect to earthquake magnitude $(M)$, source-to-site Joyner-Boore distance $(R)$, and $\varepsilon\left(T_{1}\right)$. We calculate $\varepsilon\left(T_{1}\right)$ using the GMPEs developed by Boore and Atkinson (2008). We measure the sufficiency of $\mathrm{Sa}^{70 \%}\left(1.5 T_{1}\right)$ instead of that of $\mathrm{Sa}^{70 \%}\left(T_{1}\right)$ or $\mathrm{Sa}^{70 \%}\left(2 T_{1}\right)$ because it generally is the more efficient IM for the four EDPs representing highly nonlinear response: $\mathrm{MIDR}=0.03$, $\mathrm{MIDR}=0.06, \mathrm{MIDR}=0.1$, and collapse. Furthermore, the sufficiency statistics for $S a^{70 \%}\left(1.5 T_{1}\right)$ are generally similar to those of $S a^{70 \%}\left(T_{1}\right)$ or $S a^{70 \%}\left(2 T_{1}\right)$. We compare the sufficiency of $S a^{70 \%}\left(1.5 T_{1}\right)$ with that of $S a^{5 \%}\left(T_{1}\right), S a_{\text {avg }}^{2 T_{1}}$, and $S a_{\text {avg }}^{3 T_{1}}$.

To evaluate sufficiency, we use the framework implemented by Luco and Cornell (2007) and Eads et al. (2015). For each building model, IM, EDP, and ground motion characteristic $\left[M, R\right.$, and $\left.\varepsilon\left(T_{1}\right)\right]$, we perform a linear regression of the following form:

$$
\log (I M)=\beta_{0}+\beta_{1} \times x
$$

where $x=M, \log (R)$, or $\varepsilon\left(T_{1}\right)$. Here, $\log (R)$ is the base-10 logarithm of $R$. Each regression is conducted on a set of 50 data points representing the IM values that induce the given EDP in the given building model for the set of 50 ground motions. If an IM is perfectly sufficient, then we would expect $\beta_{1}=0$, indicating no dependence of the IM values on the given ground motion characteristic. For each linear regression, we thus perform a hypothesis test for which the null hypothesis is $\beta_{1}=0$ and calculate the corresponding $p$-value. The $p$-value represents the probability that $\beta_{1}$ calculated from regression could be observed if the true value is $\beta_{1}=0$. We use a $5 \%$ significance level to judge sufficiency. That is, if the $p$-value for a regression is greater than 0.05 , then we say the given IM is sufficient with respect to the given ground motion characteristic for the specific building model and EDP.

In addition to the $p$-value, we calculate the correlation coefficient, $\rho$, for each regression. For a given linear regression, $\rho=1$ indicates a perfect positive linear relationship (i.e., slope is positive), $\rho=-1$ indicates a perfect negative linear relationship (i.e., slope is negative), 
and $\rho=0$ indicates no linear relationship (i.e., slope is zero). As such, $\rho$ close to zero indicates that sufficiency is likely, while $\rho$ far from zero indicates that sufficiency is unlikely. Therefore, for a given regression, if the $p$-value is small, then $\rho$ is far from zero, and if the $p$-value is large, then $\rho$ is close to zero. The $p$-value provides a quantitative definition for sufficiency (i.e., 5\% significance level), while $\rho$ represents the nature of the correlation between the IM and ground motion characteristic (i.e., positive or negative correlation).

To demonstrate this procedure and how the $p$-value and $\rho$ are obtained for a given regression, Figure 3 shows scatter plots of $\ln \left[S a^{5 \%}\left(T_{1}\right)\right]$ versus $M$ and $\ln \left[S a^{5 \%}\left(T_{1}\right)\right]$ versus $\varepsilon\left(T_{1}\right)$ for MIDR $=0.06$ for the 9P-85 model. In this example, $S a^{5 \%}\left(T_{1}\right)$ is insufficient with respect to $\varepsilon\left(T_{1}\right)$ for MIDR $=0.06$ for the $9 \mathrm{P}-85$ model because the $p$-value is less than 0.05 . Note that even though the $p$-value is only 0.002 for $S a^{5 \%}\left(T_{1}\right)$ with respect to $\varepsilon\left(T_{1}\right)$, there is significant scatter in the regression as indicated by the fact that $\rho=0.425$ (i.e., not close to 1 ).

Tables 5 and 6 show the percent of $\mathrm{P}$ and B models, respectively, that are sufficient for $S a^{5 \%}\left(T_{1}\right), S a^{70 \%}\left(1.5 T_{1}\right), S a_{\text {avg }}^{2 T_{1}}$, and $S a_{\text {avg }}^{3 T_{1}}$ with respect to $M, \log (R)$, and $\varepsilon\left(T_{1}\right)$ for the four

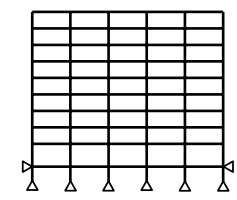

$$
\begin{gathered}
\text { 9P-85 model } \\
T_{1}=2.16 \mathrm{~s} \\
M I D R=0.06
\end{gathered}
$$

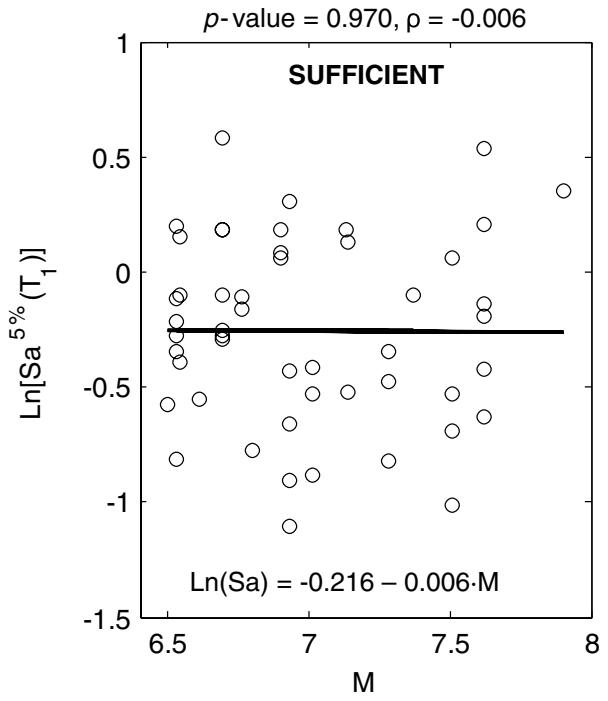

(a)

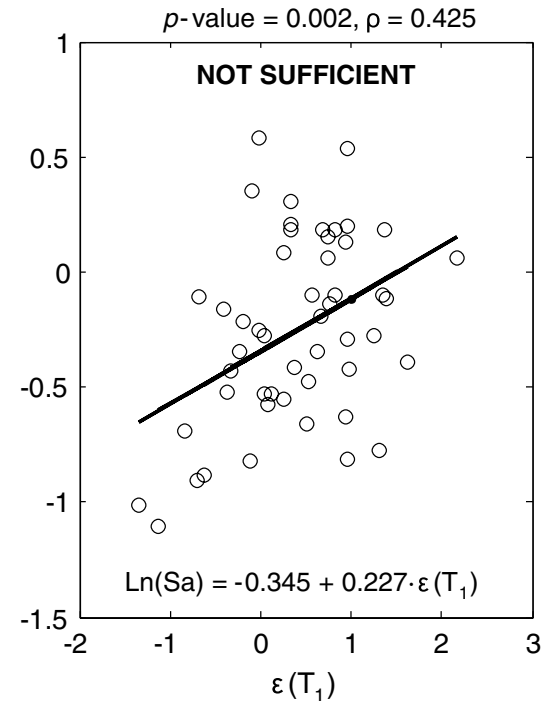

(b)

Figure 3. Demonstration of the data from which $p$-values and $\rho$ for a given IM with respect to a given ground motion characteristic are calculated for a given building model and EDP. In this example, $p$-values and $\rho$ for $S a^{5 \%}\left(T_{1}\right)$ with respect to (a) $M$ and (b) $\varepsilon\left(T_{1}\right)$ are calculated for the 9P-85 model and MIDR $=0.06$. In this case, $S a^{5 \%}\left(T_{1}\right)$ is sufficient with respect to $M$ and insufficient with respect to $\varepsilon\left(T_{1}\right)$ if a $5 \%$ significance level $(p$-value $=0.05)$ is used to judge sufficiency. 
Table 5. Percent of $\mathrm{P}$ models for which the $p$-value $\geq 0.05$ for the four considered $\mathrm{EDPs}(\mathrm{MIDR}=0.03, \mathrm{MIDR}=0.06, \mathrm{MIDR}=0.1$, and collapse)

\begin{tabular}{lcccc}
\hline \hline & \multicolumn{4}{c}{$\%$ of considered responses with $p$-value $\geq 0.05$} \\
\cline { 2 - 5 } Parameter & $S a^{5 \%}\left(T_{1}\right)$ & $S a^{70 \%}\left(1.5 T_{1}\right)$ & $S a_{\text {avg }}^{2 T_{1}}$ & $S a_{\text {avg }}^{3 T_{1}}$ \\
\hline$M$ & 95 & 93 & 89 & 73 \\
$\log (R)$ & 98 & 95 & 86 & 82 \\
$\varepsilon\left(T_{1}\right)$ & 5 & 77 & 50 & 48 \\
\hline \hline
\end{tabular}

Note: Calculated percentages consider 44 total responses (11 models, four EDPs).

Table 6. Percent of $\mathrm{B}$ models for which the $p$-value $\geq 0.05$ for the four considered EDPs (MIDR $=0.03, \mathrm{MIDR}=0.06, \mathrm{MIDR}=0.1$, and collapse)

\begin{tabular}{lcccc}
\hline \hline & \multicolumn{4}{c}{$\%$ of considered responses with $p$-value $\geq 0.05$} \\
\cline { 2 - 5 } Parameter & $S a^{5 \%}\left(T_{1}\right)$ & $S a^{70 \%}\left(1.5 T_{1}\right)$ & $S a_{\mathrm{avg}}^{2 T_{1}}$ & $S a_{\text {avg }}^{3 T_{1}}$ \\
\hline$M$ & 93 & 82 & 100 & 84 \\
$\log (R)$ & 70 & 55 & 80 & 84 \\
$\varepsilon\left(T_{1}\right)$ & 25 & 84 & 61 & 59 \\
\hline \hline
\end{tabular}

Note: Calculated percentages consider 44 total responses (11 models, four EDPs).

considered EDPs. Statistics for the four EDPs are combined in these tables, so each percent represents the fraction out of 44 total $p$-values (11 building models and four EDPs). Note that by using a 5\% significance level to define sufficiency, we expect that about $5 \%$ of calculated $p$-values will be less than 0.05 even if the IM is completely sufficient with respect to the considered ground motion parameter. That is, if an IM is completely sufficient, we would expect the reported value in Tables 5 and 6 to be $95 \%$ or greater. For brevity, the $p$-values and $\rho$ calculated for each regression are not shown here but are available in appendix B of Buyco (2018) for $S a^{5 \%}\left(T_{1}\right), S a^{70 \%}\left(1.5 T_{1}\right)$, and $S a_{\text {avg }}^{3 T_{1}}$.

It appears that all four IMs are usually sufficient with respect to $M$. Even though Eads et al. (2015) also found $S a^{5 \%}\left(T_{1}\right)$ and $S a_{\text {avg }}^{3 T_{1}}$ to be sufficient with respect to $M$ for evaluating the collapse of steel moment frame building models, some caution should be exercised when interpreting these results. As observed by Eads et al. (2015), sufficiency with respect to $M$ can be improperly classified by the $p$-value if the range of $M$ values in the input ground motions is not large enough. In this study, $M$ spans a range of $6.50 \leq M \leq 7.90$, so records from small magnitude events are not included. This may result in classifying an IM as sufficient with respect to $M$, while increasing the range of earthquake magnitudes would result in classifying the IM as insufficient. There is also significant scatter in the data when sufficiency with respect to $M$ is evaluated, which can be seen visually in Figure 3a. As such, a slight dependence of an IM on $M$ may be lost in the record-to-record variability. The results presented in Tables 5 and 6 should therefore be interpreted to show that none of the considered IMs are 
dramatically insufficient with respect to $M$, but a slight dependence on $M$ should not be ruled out.

It is worth discussing further the apparent sufficiency of $S a^{5 \%}\left(T_{1}\right)$ with respect to $M$, particularly for the 20-story models for which higher-mode response would seem to be important. Some have found $S a^{5 \%}\left(T_{1}\right)$ to be sufficient with respect to $M$ for predicting interstory drift ratios (e.g., Bradley et al. 2010, Aslani and Miranda 2005) of building models with 10 or fewer stories, but others (e.g., Luco and Cornell 2007, Kazanti and Vamvatsikos 2015) have concluded the opposite. Luco and Cornell found $S a^{5 \%}\left(T_{1}\right)$ to be insufficient with respect to $M$ for steel moment frame models with heights ranging from 3 to 20 stories, which seems to contradict the results found in this study. One possible explanation for this discrepancy is that our study focuses on MIDR $\geq 0.03$, while most of the responses considered by Luco and Cornell have MIDR $<0.03$. Kazanti and Vamvatsikos, however, found $S a^{5 \%}\left(T_{1}\right)$ to be insufficient with respect to $M$ for MIDR $>0.03$ for mid- and high-rise reinforced-concrete (RC) frame models. There is not an obvious explanation for why these results are different than those in our study, but it is possible that sufficiency evaluations may be different for steel and RC frame structures. For example, Eads et al. (2015) found $S a^{5 \%}\left(T_{1}\right)$ to be sufficient with respect to $M$ for nearly all steel moment frame models but for only $20 \%$ of RC moment frame models, though different sets of ground motions were used in these evaluations. Further work should be done to understand these differences.

With respect to $\log (R)$, all four IMs are almost always sufficient for the P models. Sufficiency with respect to $\log (R)$ is also observed in most cases by Eads et al. (2015) for $S a^{5 \%}\left(T_{1}\right)$ and $S a_{\text {avg }}^{3 T_{1}}$. For the B models, $S a^{70 \%}\left(1.5 T_{1}\right)$ is usually sufficient but is less likely to be sufficient than $S a^{5 \%}\left(T_{1}\right), S a_{\text {avg }}^{2 T_{1}}$, and $S a_{\text {avg }}^{3 T_{1}}$. Most of the regressions that result in insufficiency for $S a^{70 \%}\left(1.5 T_{1}\right)$ are for MIDR $=0.1$ and collapse. The values of $\rho$ for $S a^{70 \%}\left(1.5 T_{1}\right)$ with respect to $\log (R)$ for the $\mathrm{B}$ models are usually less than zero, indicating that for a given value of $S a^{70 \%}\left(1.5 T_{1}\right)$, a ground motion with small $R$ is likely to induce a less severe response in a B model than a ground motion with large $R$. The same observation regarding $\rho$ can be made about $S a^{5 \%}\left(T_{1}\right)$. For $S a_{\text {avg }}, \rho$ is usually negative for the 3B and 9B models but usually positive for the 20B models. There is not an obvious explanation for these observations, but because all the IMs are usually sufficient with respect to $\log (R)$ for all B models, it is not an immediate concern. However, that these observations are specific to the B models may warrant investigation in future work.

Based on the results shown in Tables 5 and 6, under the considered framework for evaluating sufficiency, all four IMs are usually sufficient with respect to $M$ and $\log (R)$, but we found important differences among the IMs with respect to $\varepsilon\left(T_{1}\right)$, which warrants further discussion. Consistent with the observations of Eads et al. (2015), $S a^{5 \%}\left(T_{1}\right)$ is rarely sufficient with respect to $\varepsilon\left(T_{1}\right)$, while $S a_{\text {avg }}^{2 T_{1}}$ and $S a_{\text {avg }}^{3 T_{1}}$ are sufficient with respect to $\varepsilon\left(T_{1}\right)$ in about half of cases. $S a^{70 \%}\left(1.5 T_{1}\right)$ is sufficient in $77 \%$ of the considered responses for the P models and $84 \%$ of the considered responses for the B models with respect to $\varepsilon\left(T_{1}\right)$. Thus for both sets of models, with respect to $\varepsilon\left(T_{1}\right), S a^{70 \%}\left(1.5 T_{1}\right)$ is sufficient in slightly more cases than $S a_{\text {avg }}^{2 T_{1}}$ and $S a_{\text {avg }}^{3 T_{1}}$, and all three of these advanced IMs are much more likely to be sufficient than $\mathrm{Sa}^{5 \%}\left(T_{1}\right)$. For $S a^{5 \%}\left(T_{1}\right), \rho>0$ in every regression. This is not surprising because ground motions with large $\varepsilon\left(T_{1}\right)$ tend to have a less severe spectral shape (Baker and Cornell 2005). For $S a^{70 \%}\left(1.5 T_{1}\right), S a_{\text {avg }}^{2 T_{1}}$, and $S a_{\text {avg }}^{3 T_{1}}, \rho$ is usually close to zero for MIDR $=0.03$ but is 


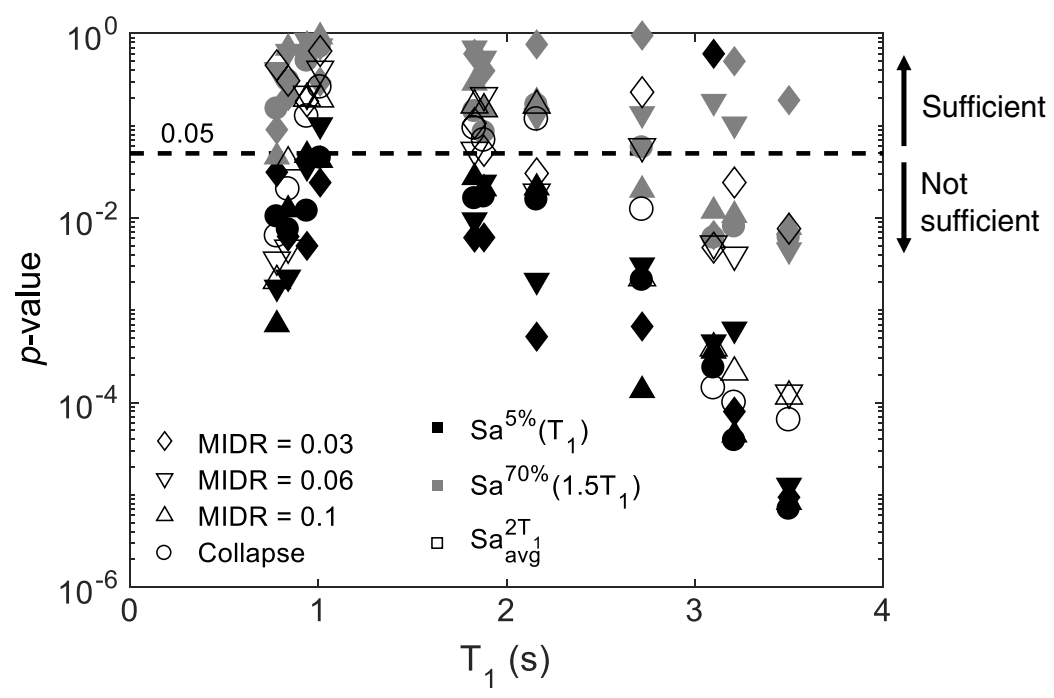

Figure 4. The $p$-values from evaluating sufficiency of $S a^{5 \%}\left(T_{1}\right), S a^{70 \%}\left(1.5 T_{1}\right)$, and $S a_{\text {avg }}^{2 T_{1}}$ with respect to $\varepsilon\left(T_{1}\right)$ for all P models and four EDPs: MIDR $=0.03$, MIDR $=0.06, \operatorname{MIDR}=0.1$, and collapse.

almost always greater than zero for $\mathrm{MIDR}=0.06, \mathrm{MIDR}=0.1$, and collapse. This is generally true for all building models.

To further demonstrate the trends in sufficiency with respect to $\varepsilon\left(T_{1}\right)$, Figure 4 plots the associated $p$-values as a function of $T_{1}$ of the $\mathrm{P}$ models for $S a^{70 \%}\left(1.5 T_{1}\right), S a^{5 \%}\left(T_{1}\right)$, and $S a_{\text {avg }}^{2 T_{1}}$. The $p$-values for $S a_{\text {avg }}^{3 T_{1}}$ are not shown, as they are generally similar to those of $S a_{\text {avg }}^{2 T_{1}}$. Each data point represents a different building model and EDP. For all IMs, the $p$-values tend to decrease as $T_{1}$ gets larger, indicating that the considered IMs are less likely to be sufficient for taller buildings for which higher-mode response may be a factor. When the IM is $\mathrm{Sa}^{70 \%}\left(1.5 T_{1}\right)$ for $\mathrm{P}$ models with $T_{1}<2.5 \mathrm{~s}, 96 \%$ of the considered responses are sufficient with respect to $\varepsilon\left(T_{1}\right)$.

\section{COMPARISON BETWEEN $S a^{70 \%}(T)$ AND $S a_{\text {avg }}$}

As previously discussed, Tables 2-4 demonstrate that the efficiency of $\mathrm{Sa}^{70 \%}(T)$ and $S a_{\text {avg }}$ for EDPs representing a highly nonlinear response are relatively similar, with the exact level of efficiency dependent on $T$ for $S a^{70 \%}(T)$, the period range for $S a_{\text {avg }}$, and the EDP of interest. $S a_{\text {avg }}$ is also similar to $S a^{70 \%}(T)$ in terms of what it measures about a ground motion, and this similarity necessitates further discussion. These IMs are alike in that they both measure the extent to which a broad range of periods are present in a ground motion, with an emphasis on long periods. Here, the "range of periods present in a ground motion" refers to a range of periods in the Fourier decomposition of the ground motion, not the response spectrum, which is an important distinction.

To demonstrate the long-period emphasis of $S a_{\text {avg }}$ and $S a^{70 \%}(T)$, consider the case in which the ground motion acceleration is a simple harmonic with angular frequency $\omega_{g}$ and 
unit amplitude. That is, $\ddot{u}_{g}(t)=\cos \left(\omega_{g} t\right)$. In this case, the steady-state response of an SDOF with period $T_{s}$ and damping ratio $\zeta_{s}$ is $u_{s s}(t)=U_{s s} \cos \left(\omega_{g} t+\delta\right)$, where $\delta$ is the phase difference between $u_{s s}(t)$ and $\ddot{u}_{g}(t)$, and $U_{s s}$ is given by:

$$
U_{s s}=\left[\left(\omega_{s}^{2}-\omega_{g}^{2}\right)^{2}+\left(2 \zeta_{s} \omega_{s} \omega_{g}\right)^{2}\right]^{-1 / 2} .
$$

Plots of $\omega_{s}^{2} U_{s s}$ versus $T_{g} / T$ are shown in Figure 5 for an SDOF with $T_{s}=T$ and $\zeta_{s}=5 \%$, an SDOF with $T_{s}=T$ and $\zeta_{s}=70 \%$, and an SDOF with $T_{s} \rightarrow 0$ and $\zeta_{s}=5 \%$. Note that $T_{g}=2 \pi / \omega_{g}$. In the same figure, $\omega_{s}^{2} U_{s s}$ is plotted for SDOFs with $\zeta_{s}=5 \%$ and $T_{s}$ ranging from $0.2 T$ to $2 T$ spaced arithmetically $(0.1 \mathrm{sec}$. spacing $)$ to represent the responses that are used to calculate $S a_{\mathrm{avg}}^{2 T}$. The geometric mean of these 5\%-damped spectra is plotted to approximately represent $S a_{\mathrm{avg}}^{2 T}$.

Figure 5 shows the degree to which different periods in a ground motion contribute to the calculations of $S a^{5 \%}(T), S a_{\mathrm{avg}}^{2 T}$, and $S a^{70 \%}(T)$. The resonance peak in the curve of $S a^{5 \%}(T)$ around $T_{g}=T$ means that when $S a^{5 \%}(T)$ for a ground motion is calculated, the ground motion's spectral content around a period of $T$ is heavily weighted. In comparison to $S a^{5 \%}(T)$, the curve of $S a_{\mathrm{avg}}^{2 T}$ has a lower and wider peak around $T_{g}=2 T$, indicating that spectral content from a ground motion in the range of $T$ to $3 T$ is most heavily weighted when calculating $S a_{\text {avg }}^{2 T}$.

The curve of $S a^{5 \%}\left(T_{s} \rightarrow 0\right)$ is equal to one at all periods. For a given ground motion, $S a^{5 \%}\left(T_{s} \rightarrow 0\right) \approx$ PGA, so the purpose of the curve of $S a^{5 \%}\left(T_{s} \rightarrow 0\right)$ is to demonstrate how

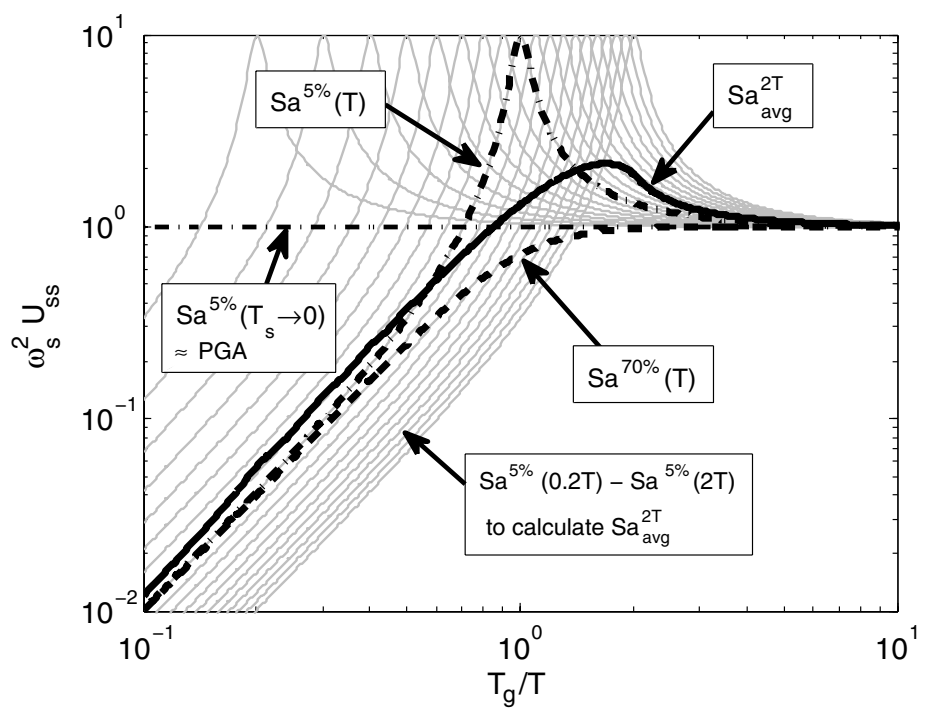

Figure 5. Steady-state response $U_{s s}$ of SDOFs with period $T_{s}=T=2 \pi / \omega$ and $\zeta=5 \%$ and $70 \%$ to harmonic acceleration excitation with period $T_{g}$ and unit amplitude. The steady-state response of an SDOF with period $T_{s} \rightarrow 0$ and $\zeta=5 \%$ is also plotted. Also shown are steady-state responses of SDOFs with $T_{s}$ ranging from $0.2 T$ to $2 T$ and $\zeta=5 \%$, whose geometric mean is used to calculate $S a_{\mathrm{avg}}^{2 T}$. 
different IMs emphasize different periods in a ground motion relative to PGA. For example, in its calculation, $S a_{\text {avg }}^{2 T}$ gives more weight to periods in a ground motion in the range of $T$ to $3 T$ than PGA does, but the calculation of PGA gives more weight to periods less than $T$ than $S a_{\text {avg }}^{2 T}$ does.

In contrast to $S a^{5 \%}(T)$ and $S a_{\text {avg }}^{2 T} S a^{70 \%}(T)$ has no resonance peak. Instead, its corresponding steady-state response for $T_{g}<T$ increases until nearly reaching a value of one at $T_{g}=T$, which it approaches asymptotically for $T_{g}>T$. The interpretation here is that $\mathrm{Sa}^{70 \%}(T)$ is a measure of the long-period content in a ground motion with all periods longer than $T$ being given approximately equal weight, even for $T \rightarrow \infty$. Similar to $\mathrm{Sa}^{5 \%}(T)$ and $S a_{\mathrm{avg}}^{2 T}, S a^{70 \%}(T)$ gives relatively little weight to periods in a ground motion shorter than $T$. However, compared with $S a^{5 \%}(T)$, both $S a_{\text {avg }}^{2 T}$ and $S a^{70 \%}(T)$ give relatively more weight to a range of periods longer than $T$ than to periods close than $T$. As previously described, the calculation of $\mathrm{Sa}^{5 \%}(T)$ is dominated by periods close to $T$. It should be noted that the observations made here about $S a_{\mathrm{avg}}^{2 T}$ also generally apply to $S a_{\mathrm{avg}}^{1.5 T}$ and $S a_{\mathrm{avg}}^{3 T}$. Of course, $S a_{\text {avg }}$ can be calculated with any arbitrary range of periods to suit the specific application, and each distinct definition of $S a_{\text {avg }}$ will have different periods that are emphasized.

As has been demonstrated thus far, there are many similarities between $S a_{\mathrm{avg}}$ and $S a^{70 \%}(T)$ as IMs, but it is important to note their differences, particularly with respect to application in practice. Unlike $S a^{70 \%}(T)$, different versions of $S a_{\text {avg }}$ have been studied extensively, finding different period ranges that perform best for EDPs other than MIDR, such as peak floor acceleration (e.g., Kazanti and Vamvatsikos 2015). Another significant advantage of $S a_{\mathrm{avg}}$ is that direct GMPEs for $S a_{\mathrm{avg}}$ have been recently developed (Kohrangi et al. 2018), so the seismic hazard with respect to $S a_{\text {avg }}$ can be calculated, and $\lambda[I M]$ from Equation 1 can be computed to estimate risk. The hazard for $S a_{\text {avg }}$ can also be inferred indirectly from GMPEs for $S a^{5 \%}(T)$ and correlation coefficients between different periods (Baker and Jayaram 2008). In contrast, direct GMPEs do not exist for $\mathrm{Sa}^{70 \%}(T)$, and indirect hazard cannot be inferred because $\mathrm{Sa}^{70 \%}(T)$ is not calculated from other IMs. Of course, the existence of GMPEs is not an inherent characteristic of an IM. Nonetheless, the development of GMPEs is a significant barrier to advanced IMs being used in practice.

One potential advantage of $\mathrm{Sa}^{70 \%}(T)$ is its equivalence to the peak acceleration of a ground motion after a second-order low-pass Butterworth filter has been applied. That is, $S a^{70 \%}(T)$ measures the peak long-period acceleration of a ground motion, a physically meaningful quantity. In some cases, this may provide an avenue for using $\mathrm{Sa}^{70 \%}(T)$ to qualitatively estimate a ground motion's potential to induce a highly nonlinear response. Suppose a ground motion is dominated by long-period content at much longer periods than $T_{1}$ of the building of interest. This could occur for a pulse-like ground motion that has a longduration pulse. In such a case, the building may respond in a quasistatic manner to the longperiod shaking. The capacity of a building to withstand quasistatic excitation can be estimated by pushover analysis, which provides $V_{\max } / M$, approximately the maximum quasistatic acceleration that the building can resist without collapsing. Here, $M$ is the building model's seismic mass.

Song and Heaton (2012) predict collapse of a building model by directly comparing the peak of a ground motion's low-pass filtered acceleration to $V_{\max } / M$. In Song and Heaton's collapse prediction framework, the filter's corner period is defined to be $c T_{1}$, where $c$ is some 
coefficient (usually between 1 and 2) dependent on the building model's ductility. To replicate the framework of Song and Heaton, we find the sets of 50 scaled ground motions for each building model for which $\mathrm{Sa}^{70 \%}(T)$ first exceeds $V_{\max } / M$ for $T=T_{1}, 1.5 T_{1}$, and $2 T_{1}$. We compute the MIDR induced in the building model for each set of 50 scaled ground motions and combine the statistics for each set of building models. From the combined statistics, we calculate the median MIDR and associated "pseudodispersion," $\hat{\sigma}_{\text {In }}$, for each set of models, where $\hat{\sigma}_{\text {ln }}$ is one-half the difference between the 84 th and 16th percentile natural logarithms of the MIDRs. We compute the median and $\hat{\sigma}_{\text {ln }}$ to estimate the geometric mean and $\sigma_{\text {In }}$ as some of these scaled ground motions induce collapse in the building models, which introduce values of infinite MIDR into the data set. In some cases, more than $16 \%$ of scaled ground motions induce collapse, so $\hat{\sigma}_{\text {ln }}$ is infinite. In these situations, we calculate $\hat{\sigma}_{\text {ln }}$ as the difference between the median and 16th percentile natural logarithms of the MIDRs and indicate $\hat{\sigma}_{\text {ln }}$ with an asterisk. The results are shown in Table 7.

In Table 7, the median MIDR of all building model sets are qualitatively in the range of 0.03 if $S a^{70 \%}\left(T_{1}\right)=V_{\max } / M$, in the range of 0.04 if $S a^{70 \%}\left(1.5 T_{1}\right)=V_{\max } / M$, and in the range of 0.06 or greater if $S a^{70 \%}\left(2 T_{1}\right)=V_{\max } / M$. Interestingly, these trends generally hold across all building model sets; however, the median MIDRs tend to be slightly smaller for the taller buildings and the median response if $\operatorname{Sa}^{70 \%}\left(2 T_{1}\right)=V_{\max } / M$ for the $20 \mathrm{~B}$ models is "collapse" because the 20B models cannot withstand MIDRs much greater than 0.06 without collapsing. $\hat{\sigma}_{\mathrm{ln}}$ for the $\mathrm{P}$ models are smaller than those for the $\mathrm{B}$ models, which is to be expected because each $\mathrm{B}$ model has its weld fracture strain assigned according to a random distribution.

There is some scatter in the results shown in Table 7, but there appears to be some promise regarding comparing $S a^{70 \%}(T)$ to $V_{\max } / M$. In particular, if $T$ is chosen between $T_{1}$ and $2 T_{1}$, then if $S a^{70 \%}(T)$ for a ground motion is greater than $V_{\max } / M$ for the building model, highly nonlinear response seems likely. Song and Heaton (2012) address the importance of ductility and of ground motion type when making the comparison of peak low-pass filtered acceleration to $V_{\max } / M$, but more work should be done to clarify when this analysis is appropriate. Nonetheless, this potential physical interpretation of $\mathrm{Sa}^{70 \%}(T)$ may offer an advantage

Table 7. Median and $\hat{\sigma}_{\ln }$ of MIDRs induced in each set of building models when $\operatorname{Sa}^{70 \%}(T)=$ $V_{\max } / M$ for each building model

\begin{tabular}{|c|c|c|c|c|c|c|}
\hline \multirow[b]{2}{*}{ Model set } & \multicolumn{2}{|l|}{$T=T_{1}$} & \multicolumn{2}{|c|}{$T=1.5 T_{1}$} & \multicolumn{2}{|c|}{$T=2 T_{1}$} \\
\hline & Median MIDR & $\hat{\sigma}_{\ln }$ & Median MIDR & $\hat{\sigma}_{\ln }$ & Median MIDR & $\hat{\sigma}_{\text {ln }}$ \\
\hline $3 \mathrm{P}$ & 0.033 & 0.24 & 0.045 & 0.19 & 0.058 & 0.27 \\
\hline $9 \mathrm{P}$ & 0.027 & 0.18 & 0.041 & 0.20 & 0.061 & 0.24 \\
\hline $20 \mathrm{P}$ & 0.024 & 0.27 & 0.038 & 0.21 & 0.062 & $0.26^{*}$ \\
\hline $3 \mathrm{~B}$ & 0.031 & 0.28 & 0.047 & $0.25^{*}$ & 0.074 & $0.39 *$ \\
\hline 9B & 0.023 & 0.23 & 0.036 & 0.27 & 0.063 & $0.35 *$ \\
\hline 20B & 0.022 & 0.41 & 0.033 & 0.40 & collapse & - \\
\hline
\end{tabular}

Note: $\hat{\sigma}_{\mathrm{ln}}$ is calculated as difference between median and 16th percentile natural logarithm of the MIDRs because over $16 \%$ of responses are collapses. 
over $S a_{\text {avg }}$ in that the value of $S a^{70 \%}(T)$ can represent something meaningful about the ground motion, whereas the value of $S a_{\text {avg }}$ does not generally have a physical meaning.

It should be noted that the concept of predicting the response of a nonlinear dynamic system with an equivalent linear SDOF that has a lengthened $T$ and larger $\zeta$ for applications in earthquake engineering has been well studied. A number of researchers have proposed different models (e.g., Iwan 1980, Rosenblueth and Herrera 1964, Gulkan and Sozen 1974, Kowalsky 1994), some of which are compiled and evaluated by Miranda and Ruiz-Garcia (2002). For these equivalent linear SDOFs, the goal is commonly to minimize the error between the maximum displacement during excitation of some nonlinear SDOF and the maximum displacement during excitation of the equivalent linear SDOF. In this respect, the models evaluated by Miranda and Ruiz-Garcia (2002) can be quite accurate on average, with errors usually less than $20 \%$. The most accurate models assigned damping ratios to the equivalent SDOFs in the range of $\zeta=10 \%-20 \%$, which is much less $\zeta=70 \%$. One reason for this discrepancy is that SDOFs with $\zeta=70 \%$ are not meant to necessarily be "equivalent" linear SDOFs to the building models such that their maximum deformations are directly related. Instead, it is simply observed in this paper that if $\mathrm{Sa}^{70 \%}(T)$ of a ground motion with $T$ between $T_{1}$ and $2 T_{1}$ exceeds $V_{\max } / M$ of the corresponding building model, this tends to be an indicator that a highly nonlinear response will occur in the building model in response to the ground motion.

\section{CONCLUSIONS}

As an IM for evaluating highly nonlinear response (MIDR $\geq 0.03$ ) of the steel moment frame building models considered in this study, $\mathrm{Sa}^{70 \%}(T)$ is generally efficient and sufficient, particularly if $T$ is chosen in the range of $T_{1}$ to $2 T_{1}$. Within this range, $T=1.5 T_{1}$ tends to work best for the considered levels of highly nonlinear response. The performance of $S a^{70 \%}(T)$ as an IM is similar to that of $S a_{\text {avg }}$ in a number of ways. The two IMs are much more likely to be sufficient with respect to $\varepsilon\left(T_{1}\right)$ than $S a^{5 \%}\left(T_{1}\right)$. Both can be tuned depending on the application $-S a^{70 \%}(T)$ by adjusting $T$, and $S a_{\text {avg }}$ by adjusting the period range. In fact, depending on the application, a different damping ratio (e.g., $50 \%$ or $100 \%$ ) may be preferable to $70 \%$ for highly damped $S a^{\zeta}(T)$. Both IMs are forgiving in terms of the estimation of $T_{1}$, as small inaccuracies in $T_{1}$ do not have a significant impact on either efficiency or sufficiency. Both IMs also tend to emphasize a wide range of periods longer than $T_{1}$ present in the ground motion when compared with $S a^{5 \%}\left(T_{1}\right)$. The emphasized range of periods for $S a_{\text {avg }}$ can be modified to suit the particular application by modifying the range of periods in calculating the average. The same can be done for $\mathrm{Sa}^{70 \%}(T)$ by either adjusting $T$ or by using a damping ratio smaller or larger than $70 \%$.

Currently, the biggest disadvantage of using $\mathrm{Sa}^{70 \%}(T)$ as an IM, or any highly damped spectral acceleration, is that unlike $S a_{\text {avg }}$, GMPEs do not yet exist. However, this is not an intrinsic characteristic of $\mathrm{Sa}^{70 \%}(\mathrm{~T})$ but rather a matter of future research efforts. One potential benefit to $\mathrm{Sa}^{70 \%}(T)$ is that it is equivalent to the peak of a ground motion's acceleration after it has been filtered with a second-order low-pass Butterworth filter that has a corner period $T$. This provides $\mathrm{Sa}^{70 \%}(T)$ with a physical meaning, which may be useful for estimating a ground motion's potential for inducing a highly nonlinear response, though further work should to be done to clarify cases in which this physical interpretation can be beneficial. 
In addition, more work needs to be done to understand the effectiveness of $\operatorname{Sa}^{70 \%}(T)$ with EDPs other than MIDR (e.g., peak floor acceleration), with lateral force-resisting systems other than steel moment frames and with different classes of ground motions (e.g., pulse-like vs. nonpulse-like).

\section{ACKNOWLEDGMENTS}

We thank Anthony Massari for providing feedback and comments on early versions of this paper. We thank the anonymous reviewer, whose feedback greatly improved the quality of this paper. This material is based on work supported by the National Science Foundation Graduate Research Fellowship Program under Grant No. DGE-1144469. Any opinions, findings, and conclusions or recommendations expressed in this material are those of the authors and do not necessarily reflect the views of the National Science Foundation.

\section{REFERENCES}

Akkar, S., and Ozen, O., 2005. Effect of peak ground velocity on deformation demands for SDOF systems, Earthquake Engineering \& Structural Dynamics 34, 1551-1571.

American Institute of Steel Construction, 2010. Prequalified Connections for Special and Intermediate Steel Moment Frames for Seismic Applicaions, ANSI/AISC 358-10, Chicago, IL.

American Society of Civil Engineers, 2010. Minimum Design Loads for Buildings and Other Structures, ASCE/SEI 7-10, Reston, VA.

American Society of Civil Engineers, 2016. Minimum Design Loads for Buildings and Other Structures, ASCE/SEI 7-16, Reston, VA.

Applied Technology Council, 1996. Seismic Evaluation and Retrofit of Concrete Buildings, Tech. Rep. ATC-40, Redwood City, CA.

Aslani, H., and Miranda, E., 2005. Probabilistic Earthquake Loss Estimation and Loss Disagregation in Buildings, Tech. Rep. 157, John A. Blume Earthquake Engineering Center, Stanford University, Stanford, CA.

Baker, J. W., and Cornell, C. A., 2005. A vector-valued ground motion intensity measure consisting of spectral acceleration and epsilon, Earthquake Engineering \& Structural Dynamics 34, 1193-1217.

Baker, J. W., and Jayaram, N., 2008. Correlation of spectral acceleration values from NGA ground motion models, Earthquake Spectra 24, 299-317.

Bianchini, M., Diotallevi, P., and Baker, J. W., 2009. Prediction of inelastic structural response using an average of spectral accelerations, in Proceedings, 10th International Conference on Structural Safety and Reliability (ICOSSAR09), Osaka, Japan.

Boore, D. M., and Atkinson, G. M., 2008. Ground-motion prediction equations for the average horizontal component of PGA, PGV, and 5 and 10.0 s, Earthquake Spectra 24, 99-138.

Bradley, B. A., Dhakal, R. P., MacRae, G. A., and Cubrinovski, M., 2010. Prediction of spatially distributed seismic demands in specific structures: Ground motion and structural response, Earthquake Engineering \& Structural Dynamics 39, 501-520.

Buyco, K., 2018. Improving Seismic Collapse Risk Assessments of Steel Moment Frame Buildings, Ph.D. Thesis, California Institute of Technology, Pasadena, CA.

Challa, V. R. M., 1992. Nonlinear Seismic Behavior of Steel Planar Moment-Resisting Frames, Ph.D. Thesis, California Institute of Technology, Pasadena, CA. 
Challa, V. R. M., and Hall, J. F., 1994. Earthquake collapse analysis of steel frames, Earthquake Engineering \& Structural Dynamics 23, 1199-1218.

Cordova, P. P., Deierlein, G. G., Mehanny, S. S. F., and Cornell, C. A., 2000. Development of a two-parameter seismic intensity measure and probabilistic assessment procedure, In The Second US-Japan Workshop on Performance-Based Earthquake Engineering Methodology for Reinforced Concrete Building Structures, Sapporo, Japan.

Eads, L., Miranda, E., and Lignos, D. G., 2015. Average spectral acceleration as an intensity measure for collapse risk assessment, Earthquake Engineering \& Structural Dynamics 44, 2057-2073.

Federal Emergency Management Agency (FEMA), 2009. Quantification of Building Seismic Performance Factors, Tech. Rep. FEMA P695, Washington, DC.

Federal Emergency Management Agency (FEMA), 2012. Seismic Performance Assessment of Buildings, Tech. Rep. FEMA P-58, Washington, DC.

Gulkan, P., and Sozen, M., 1974. Inelastic response of reinforced concrete structures to earthquake motions, ACI Journal 71, 604-610.

Gupta, A., and Krawinkler, H., 1999. Seismic Demands for Performance Evaluation of Steel Moment Resisting Frame Structures, Tech. Rep. 132, John A. Blume Earthquake Engineering Center, Stanford University, Stanford, CA.

Guyader, A. C., 2004. A Statistical Approach to Equivalent Linearization with Application to Performance-Based Engineering, Tech. Rep. 2004-04, Earthquake Engineering Research Laboratory, California Institute of Technology, Pasadena, CA.

Hall, J. F., 1997. Seismic Response of Steel Moment Frame Buildings to Near-Source Ground Motions, Tech. Rep. 97-05, Earthquake Engineering Research Laboratory, California Institute of Technology, Pasadena, CA.

Hall, J. F., 1998. Seismic response of steel moment frame buildings to near-source ground motions, Earthquake Engineering \& Structural Dynamics 27, 1445-1464.

Hall, J. F., and Challa, V. R. M., 1995. Beam-column modeling, Journal of Engineering Mechanics 121, 1284-1291.

Haselton, C. B., and Baker, J. W., 2006. Ground motion intensity measures for collapse capacity prediction: Choice of optimal spectral period and effect of spectral shape, in Proceedings, 8th U.S. National Conference on Earthquake Engineering, San Francisco, CA.

Haviland, R., 1976. A Study of the Uncertainties in the Fundamental Translational Periods and Damping Values for Rearl Buildings, Tech. Rep. 5, Evaluation of Seismic Safety of Buildings, Massachusetts Institute of Technology, Cambridge, MA.

Iwan, W. D., 1980. Estimating inelastic response spectra from elastic spectra, Earthquake Engineering \& Structural Dynamics 8, 375-388.

Iwan, W. D., and Gates, N. C., 1979. The effective period and damping of a class of hysteretic structures, Earthquake Engineering and Structural Dynamics 7, 199-211.

Kazanti, A. K., and Vamvatsikos, D., 2015. Intensity measure selection for vulnerability studies of building classes, Earthquake Engineering \& Structural Dynamics 44, 2677-2694.

Kohrangi, M., Bazzurro, P., and Vamvatsikos, D., 2016. Vector and scalar IMs in structural response estimation, part ii: Building demand assessment, Earthquake Spectra 32, $1525-1543$.

Kohrangi, M., Kotha, S. R., and Bazzurro, P., 2018. Ground-motion models for average spectral acceleration in a period range: Direct and indirect methods, Bulletin of Earthquake Engineering 16, 45-65. 
Kowalsky, M. J., 1994. Displacement-Based Design-A Methodology for Seismic Demand Applied to RC Bridge Columns, Master's Thesis, University of California at San Diego, La Jolla, CA.

Krishnan, S., and Muto, M., 2012. Mechanism of collapse of tall steel moment-frame buildings under earthquake excitation, Journal of Structural Engineering 138, 1361-1387.

Lee, K., and Foutch, D., 2000. Performance Prediction and Evaluation of Steel Special Moment Frames for Seismic Loads, Tech. Rep., SAC/BD-00/25, SAC Background Document.

Los Angeles Tall Buildings Structural Design Council, 2015. An Alternative Procedure for Seismic Analysis and Design of Tall Buildings Located in the Los Angeles Region, Los Angeles, CA.

Luco, N., and Cornell, C. A., 2007. Structure-specific scalar intensity measures for near-source and ordinary earthquake ground motions, Earthquake Spectra 23, 357-392.

Masayoshi, N., Koshika, N., Kawano, K., Hirakawa, K., and Wada, A., 2012. Performance-based seismic design for high-rise buildings in Japan, International Journal of High-Rise Buildings 1, 155-167.

Miranda, E., and Ruiz-Garcia, J., 2002. Evaluation of approximate methods to estimate maximum inelastic displacement demands, Earthquake Engineering \& Structural Dynamics 31, 539-560.

Moehle, J., and Deierlein, G. G., 2004. A framework methodology for performance-based earthquake engineering, in Proceedings, 13th World Conference on Earthquake Engineering, Vancouver, BC, Canada.

Pacific Earthquake Engineering Research Center (PEER), 2013. PEER NGA-West2 Database, Pacific Earthquake Research Center, Berkeley, CA, available at http://peer.berkeley.edu/nga/ (last accessed 20 March 2017).

Rosenblueth, E., and Herrera, I., 1964. On a kind of hysteretic damping, Journal of Engineering Mechanics Division ASCE 90, 37-48.

Shome, N., Cornell, C. A., Bazzurro, P., and Carballo, J. E., 1998. Earthquakes, records, and nonlinear responses, Earthquake Spectra 14, 469-500.

Song, S., 2013. A New Ground Motion Intensity Measure, Peak Filtered Acceleration (PGA), to Estimate Collapse Vulnerability of Buildings in Earthquakes, Ph.D. Thesis, California Institute of Technology, Pasadena, CA.

Song, S., and Heaton, T. H., 2012. Predicting collapse of steel and reinforced-concrete frame buildings in different types of ground motions, in Proceedings, 15th World Conference on Earthquake Engineering, Lisbon, Portugal.

Tall Buildings Initative Guidelines Working Group, 2010. Guidelines for Performance-Based Seismic Design of Tall Buildings, Tech. Rep., PEER 2010/05, Pacific Earthuake Engineering Research Center, Berkeley, CA.

Vamvatsikos, D., and Cornell, C. A., 2002. Incremental dynamic analysis, Earthquake Engineering \& Structural Dynamics 31, 491-514.

Vamvatsikos, D., and Cornell, C. A., 2005. Developing efficient scalar and vector intensity measures for IDA capacity estimation by incorporating elastic spectral shape information, Earthquake Engineering \& Structural Dynamics 34, 1573-1600.

(Received 14 November 2017; Accepted 2 November 2018) 